\title{
CYCLES OF PARTITIONS
}

\author{
JØRGEN BRANDT
}

\begin{abstract}
An operation on the set of partitions of a number $n$ is introduced and the possible cycles are determined.
\end{abstract}

The problem to be discussed in the following has been circulating for some time. Let $S$ be a natural number and let $\lambda$ be a partition of $S$ of length $l$ with parts $\lambda_{1}$, $\lambda_{2}, \ldots, \lambda_{l}$. Define $T(\lambda)$ as the partition of $S$ with parts $\lambda_{1}-1, \lambda_{2}-1, \ldots, \lambda_{l}-1$, $l$, ignoring any zeros that might occur. Assume that $S=1+2+\cdots+N$. Then it turns out that repeated application of $T$ always yields the partition $1,2, \ldots, N$. We shall prove that this is indeed so.

If $S$ is arbitrary, repeated application of $T$ leads into a cycle of partitions since there are only a finite number of these. We want to determine all cycles corresponding to $S$. Now, a cycle of partitions is completely determined by the sequence of the consecutive lengths of the partitions in the cycle. Due to this observation we define sets $M_{n}$ by

$$
M_{n}=\left\{\sigma=\left(\sigma_{i}\right)_{i \in Z}\left|\max \sigma_{i}=n, \forall i: \sigma_{i}=\right|\left\{\sigma_{j} \mid j<i, \sigma_{j} \geq i-j\right\} \mid\right\} .
$$

A cycle of partitions then corresponds to a $\sigma \in M_{n}$, where $n$ is the maximal length of a partition in the cycle. If we define $\sigma, \tau \in M_{n}$ to be equivalent if there exists an integer $c$ such that $\sigma_{i+c}=\tau_{i}$ for all $i$, then the cycle determines a unique class in $M_{n}$. Conversely, a class in $M_{n}$ uniquely determines a cycle of partitions by regarding the $\sigma_{i}$ 's as the lengths of the partitions in a cycle.

The above discussion shows that we may find all cycles of partitions by determining the set of equivalence classes in $M_{n}$ and this is what we shall do. First, it is not hard to see that if $\sigma \in M_{n}$ then there exists a $p \in \mathbf{N}$ such that $\sigma_{i+p}=\sigma_{i}$ for all $i$. We call the smallest such $p$ the period of $\sigma$ and denote it by $p(\sigma)$.

LEMMA 1. Let $\sigma \in M_{n}$. If $\sigma_{i}=n$ then $\forall k \in \mathbf{Z}: \sigma_{i+k n}=n$.

ProOF. By definition of $M_{n}, \sigma_{i-n}=n$ since $n=\max \sigma_{i}$. Hence the lemma holds for negative $k$. Now $\sigma_{i+n p(\sigma)}=n$, so by what we just observed $\sigma_{i+n}=n$ as well and we are done. Q.E.D.

Proposition 2. Let $\sigma \in M_{n}$. Then $\forall i: \sigma_{i} \in\{n, n-1\}$.

Proof. We may assume $n>1$. Define $\sigma^{\prime}=\left(\sigma_{i}^{\prime}\right)_{i \in Z}$ by

$$
\begin{array}{ll}
\sigma_{i}^{\prime}=\sigma_{i} & \text { if } \sigma_{i}<n, \\
\sigma_{i}^{\prime}=n-1 & \text { if } \sigma_{i}=n .
\end{array}
$$

Using Lemma 1 it follows that $\sigma^{\prime} \in M_{n-1}$. We may assume $\sigma_{0}=n$. Now, for all

Received by the editors July $9,1981$.

1980 Mathematics Subject Classification. Primary 05A17.

(c) 1982 American Mathematical Society 0002-9939/81/0000-1105/\$02.00 
$i \in Z$ there exist integers $x$ and $y$ such that $i=x n+y(n-1)$. Then by Lemma 1 we obtain

$$
\sigma_{x n}^{\prime}=n-1 \Rightarrow \sigma_{i}^{\prime}=\sigma_{x n+y(n-1)}^{\prime}=n-1 .
$$

Hence $\sigma^{\prime}=(n-1)_{i \in Z}$ which implies the result about $\sigma$. Q.E.D.

COROLlary 3. $\sigma \in M_{n} \Rightarrow p(\sigma) \mid n$.

ProOF. Combine Lemma 1 with Proposition 2. Q.E.D.

Define for $\sigma \in M_{n}, S_{\sigma}=\sum_{i=1}^{n} \sigma_{i}-\left(\begin{array}{c}n \\ 2\end{array}\right)$. If we consider the cycle of partitions corresponding to $\sigma$ it is easy to see that the cycle consists of partitions of $S_{\sigma}$.

THEOREM 4. Let $\sigma \in M_{n}$ and assume $S_{\sigma}=1+2+\cdots+N$. Then $N=n$ and $M_{n}$ has just one class with sum $S_{\sigma}$, namely $\sigma=(n)_{i \in Z}$.

PROOF. Let $\sigma_{1}, \sigma_{2}, \ldots, \sigma_{n}$ consist of $a n-1$ 's and $n-a n$ 's. Then $S_{\sigma}=$ $\left(\begin{array}{c}n+1 \\ 2\end{array}\right)-a, 0 \leq a \leq n-1$. Simple arithmetic now shows that $n=N$ and $a=$ 0. Q.E.D.

Theorem 4 proves that if we start with any partition of $S=1+2+\cdots+N$ and apply $T$ a number of times then we always end with the partition $1,2, \ldots, N$ (corresponding to $\sigma=(n)_{i \in Z}$ ).

To determine all cycles for arbitrary $S$ notice that there exist unique $n$ and $a$ such that $S=\left(\begin{array}{c}n+1 \\ 2\end{array}\right)-a, 0 \leq a \leq n-1$. Let $C_{a}(n)$ denote the number of classes in $M_{n}$ for which $\sigma_{1}, \ldots, \sigma_{n}$ contain $a n-1$ 's and $n-a n$ 's. Then $C_{a}(n)$ is the number of cycles of partitions of $S$.

THEOREM 5. $C_{a}(n)=\frac{1}{n} \sum_{d \mid(n, a)} \varphi(d)\left(\begin{array}{l}n / d \\ a / d\end{array}\right)$, where $\varphi$ is Euler's function.

PROOF. If $\sigma_{1}, \ldots, \sigma_{n}$ is any sequence consisting of $a n-1$ 's and $n-a n$ 's, we define the sequence $\sigma$ by periodically extending to both sides. It is clear that $\sigma \in M_{n}$. Thus $C_{a}(n)$ is equal to the number of circular words of length $n$ with $a$ letters $n-1$, and $n-a$ letters $n$. The standard Pólya enumeration theory (see e.g. [1]) applies. If one carries out the details one obtains the formula in the theorem. Q.E.D.

As an example of how to find all cycles corresponding to a specific $S$ we consider $S=8$. Here $n=4, a=2$, so there are $C_{2}(4)=2$ cycles. The classes in $M_{4}$ corresponding to these cycles are represented by $\ldots, 4,3,4,3,4,3, \ldots$ and $\ldots, 4,4,3,3,4,4, \ldots$. Transforming this into cycles we get

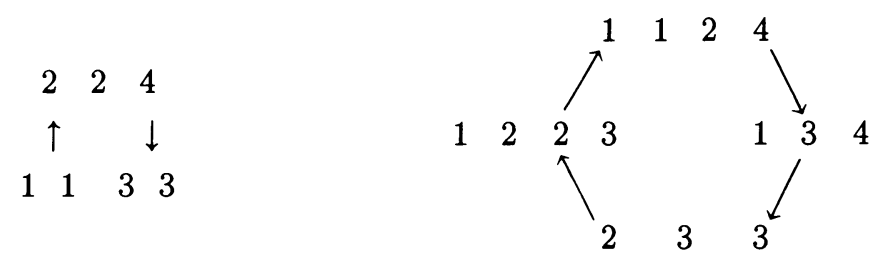

Finally we have some further comments on the special case $S=1+2+\cdots+$ $N$. Theorem 4 shows that the partitions of $S$ can be arranged in a tree so that the vertices correspond to the partitions and going down corresponds to applying $T$. The root of the tree is of course the partition $1,2, \ldots, N$. For $S=3,6$ and 10 one may draw these trees by hand but this soon becomes impracticable since the number of partitions grows rapidly. For $S=15$ there are 176 partitions. A 


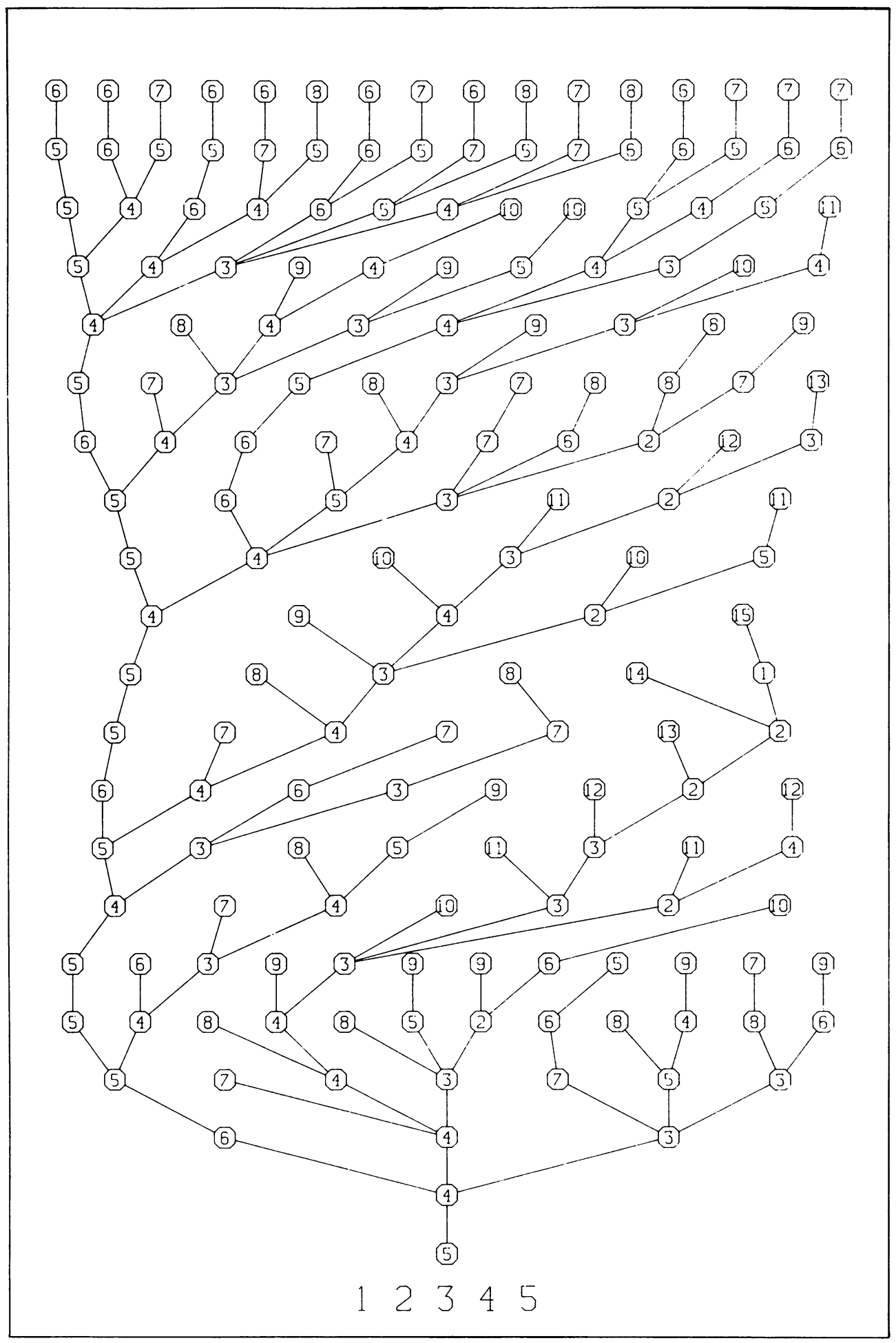

Figure 1 
computer program was written in collaboration with C. B. Hansen to generate and draw these trees. We have included the tree for $S=15$ as Figure 1 . The vertices are labeled with the length of the partitions. Notice that there are $21=5^{2}-5+1$ levels in the tree. This appears to generalize to $N^{2}-N+1$ levels in the tree for $S=1+2+\cdots+N$.

\section{REFERENCES}

1. L. L. Dornhoff and F. E. Holm, Applied modern algebra, Macmillan, New York, 1978.

MATEMAtisk INStitut, Aarhus UNIVERSitet, 8000 AARHUS, DK DANMARK 